\title{
Adaptation to suspensory locomotion in Australopithecus sediba
}

Thomas R. Rein ${ }^{\mathrm{a}, \mathrm{b}, *}$, Terry Harrison ${ }^{\mathrm{c}}$, Kristian J. Carlson ${ }^{\mathrm{d}, \mathrm{e}}$, Katerina Harvati ${ }^{\mathrm{b}}$

${ }^{a}$ Department of Anthropology, Central Connecticut State University, 1615 Stanley Street, New

Britain, CT 06053, USA

${ }^{b}$ Paleoanthropology, Senckenberg Center for Human Evolution and Paleoenvironment, Eberhard

Karls Universität Tübingen, Rümelinstr. 23, 72070 Tübingen, Germany

${ }^{c}$ Center for the Study of Human Origins, Department of Anthropology, New York University, 25

Waverly Place, New York, NY 10003, USA

${ }^{\mathrm{d}}$ Department of Cell \& Neurobiology, Keck School of Medicine, University of Southern

California, 1333 San Pablo Street, Los Angeles, CA 90033, USA

${ }^{\mathrm{e}}$ Evolutionary Studies Institute and School of Geosciences, University of the Witwatersrand,

WITS 2050, South Africa

\section{* Corresponding author.}

E-mail address: rein@ccsu.edu (T.R. Rein).

Keywords: South Africa; Ulna; Geometric morphometrics; Australopithecus afarensis; functional morphology 


\begin{abstract}
Australopithecus sediba is represented by well-preserved fossilized remains from the locality of Malapa, South Africa. Recent work has shown that the combination of features in the limb skeleton of A. sediba was distinct from that of earlier species of Australopithecus, perhaps indicating that this species moved differently. The bones of the arm and forearm indicate that $A$. sediba was adapted to suspensory and climbing behaviors. We used a geometric morphometric approach to examine ulnar shape, potentially identifying adaptations to forelimb suspensory locomotion in A. sediba. Results indicated suspensory capabilities in this species and a stronger forelimb suspensory signal than has been documented in Australopithecus afarensis. Our study confirms the adaptive significance of functional morphological traits for arboreal movements in the locomotor repertoire of $A$. sediba and provides important insight into the diversity and mosaic nature of locomotor adaptations among early hominins.
\end{abstract}




\section{Introduction}

There has been a long-standing debate regarding the nature of early hominin locomotor behavior, and much of the controversy has focused on the relative importance of arboreal locomotion in the positional repertoire of Australopithecus (e.g., Senut, 1980; Jungers, 1982; Stern and Susman, 1983; Duncan et al., 1984; Susman et al., 1984; Latimer and Lovejoy, 1990a, b; Latimer, 1991; Ohman et al., 1997; Stern, 2000; Ward, 2002; Green and Alemseged, 2012). Although features considered indicative of suspensory locomotion or climbing have been identified in the Australopithecus postcranial skeleton, the adaptive significance of these features has been questioned because it is unclear whether their occurrence is the result of stabilizing selection (i.e., they are adaptive), or because they were neither selected for nor against (i.e., they are nonaptations; Gould and Vrba, 1982; Ward, 2002). This seemingly unresolvable issue is due to the understanding that the skeletal morphology of an organism results not only from current selective pressures, but also from phylogenetic and developmental constraints imposed on it by specializations acquired earlier in its evolutionary history (e.g., Gould and Lewontin, 1979; Shubin et al., 1997; Lovejoy et al., 1999; Chiu and Hamrick, 2002; Hallgrímsson et al., 2002; Ward, 2002). Since the skeletal morphology of an organism represents a compromise between these factors, it is difficult to determine the degree to which features indicate current locomotor adaptation or reflect phylogenetic lag. Furthermore, the various species of Australopithecus have been reported to possess different mosaics of primitive and derived postcranial features, raising important questions regarding timing and the relative selective importance of skeletal modifications that accompanied the shift to obligate bipedalism in early hominins (e.g., McHenry and Berger, 1998; Green et al., 2007; Zipfel et al., 2011; DeSilva et al., 2013; Prang, 2015). 
The 1.98 million-year-old partial skeletons of Australopithecus sediba, from Malapa, South Africa, provide important insight into the positional behavior of a late surviving species of Australopithecus (Berger et al., 2010; Dirks et al., 2010; Pickering et al., 2011). The MH2 partial skeleton, identified as an adult female of this taxon, includes a number of well-preserved limb bones that have allowed researchers to infer patterns of limb use and adaptation characterizing this species. Australopithecus sediba had a hand that combined Australopithecuslike and Homo-like character states (Kivell et al., 2011), while the arm and forearm were apparently adapted to competence in climbing and suspensory locomotor behaviors (Churchill et al., 2013). Churchill and colleagues (2013) identified character states of the forelimb associated with climbing and suspension, such as a keeled ulnar trochlear notch, cranially-oriented glenoid fossa of the scapula, and high brachial index, which led them to infer that A. sediba was adapted for climbing and suspensory behaviors. Moreover, the unique combination of primitive and derived pedal features that distinguish $A$. sediba from earlier members of the genus, such as Australopithecus afarensis, have been interpreted as indicating kinematically-distinct forms of bipedalism (Zipfel et al., 2011; DeSilva et al., 2013; Prang, 2015). The preservation of complete bones offers the opportunity to examine a larger range of primitive and derived skeletal traits preserved in these fossils than would be possible in more fragmentary remains.

Among these complete long bones, the MH2 partial skeleton includes a well-preserved ulna. Previous research has demonstrated that measurements incorporating both proximal and distal regions of the ulna correspond to the extent of forelimb suspensory locomotion (FSL) performed by hominoids, arboreal cercopithecoids, and platyrrhines (Rein et al., 2015). For example, Rein et al. (2015) reported a strong relationship $\left(\mathrm{R}^{2}=0.91\right.$ in multiple regression analysis) between mean proportions of FSL and the first two principal components of mean species shape variation 
for the complete ulna. This relationship was driven mainly by the length of the ulna relative to the size of the proximal and distal joints, with suspensory species having relatively longer ulnae. However, more subtle aspects of joint shape also contributed to separating taxa that perform substantial proportions of FSL from closely related species. As in other taxa, hominids (i.e., great apes and humans) that perform substantially greater proportions of FSL were characterized, on average, by PC scores closer to those of Hylobates, a highly specialized brachiator, than other hominids. Thus, the shape of the complete ulna provides a strong adaptive signal related to brachiation and arm swing locomotion across anthropoid primates, including hominids.

The complete ulna (U.W. 88-62; Fig. 1) of the MH2 skeleton, therefore, offers the opportunity for additional insight into the adaptive significance of forelimb suspensory behaviors in A. sediba. Furthermore, the complete ulna from the A.L. 438-1 (Drapeau et al., 2005) partial skeleton of the earlier taxon A. afarensis, dating to $~ 3.0 \mathrm{Ma}$, allows direct comparison between these two hominin species, to the extent that these specimens are representative of the respective species, thus enabling assessment of the relative importance of forelimb-dominated suspension in their respective locomotor repertoires.

We employed 3D landmark measurements and traditional interlandmark distances to assess adaptation to FSL in the ulnae of $A$. sediba and A. afarensis in the context of a diverse comparative sample of anthropoid primates. The present study will test the hypothesis put forth by Churchill et al. (2013) that $A$. sediba had a forelimb adapted for substantial suspensory ability. Our geometric morphometric approach provides a comprehensive assessment of ulnar shape that builds on the analysis presented by Churchill et al (2013). The adaptive significance of FSL in A. sediba will be compared directly with that of A. afarensis, a species that has been the subject of studies on locomotor function for decades. The broad comparative context characterizing the 
present study allowed us to examine how shape related to FSL when size and phylogeny were taken into account. If the ulna of $A$. sediba is adapted to forelimb suspensory movements, it is expected that the $\mathrm{MH} 2$ fossil will display similarities in ulnar articular surface configurations with extant primates characterized by locomotor repertoires that include FSL behaviors. Furthermore, the comparison of the shape configurations of A. sediba and A. afarensis has the potential to provide insight into the selection pressures faced by these hominin species that existed at different times and were characterized by varying combinations of primitive and derived skeletal features.

\section{Materials and methods}

Extant species employed in the examination of fossil hominin ulnar shape included four platyrrhines, five hominoids, and 10 cercopithecoids (Table 1). Previously published proportion data for each species employed in regression analyses are reported in Table 1. For extant taxa, three-dimensional (3D) coordinates for 17 landmarks were collected on the proximal and distal ends of each ulna using an Immersion Microscribe digitizer (Table 2). Since there is minor erosion on the surface of the anconeal beak and inferior portion of the radial notch of the A.L. 438-1 ulna (Drapeau, 2008), analyses were performed on 17-landmark configurations that did not include landmarks on the damaged regions (as opposed to the 19-landmark analyses reported by Rein et al. [2015]). Linear measurements were collected with digital calipers (Mitutoyo Corporation) or extracted from coordinate data collected with the Microscribe digitizer based on landmarks described by Drapeau (2004). Measurements on the A.L. 438-1 left ulna were made directly on the original fossil by TRR using a Microscribe digitizer and digital calipers.

Measurements for A. sediba were collected by TRR on a surface rendering of the reconstructed 
complete right ulna of MH2 (U.W. 88-62) using Avizo 8 (Visualization Sciences Group) at the Paleoanthropology Imaging Laboratory, University of Tübingen. Cracks in the original specimen, for example in the trochlear notch, were removed by KJC during the reconstruction. The process involved segmenting and separating parts bordering the cracks, then repositioning individual parts in anatomical orientation. When possible, internal structure (e.g., trabecular struts) was used to align conjoining parts in three (orthogonal) directions. This procedure was performed in VGStudio Max 2.1 (Volume Graphics GmbH, Heidelberg, Germany).

We processed complete ulnar landmark coordinate configurations representing mean species shape using generalized Procrustes analysis (GPA; Gower, 1975; Rohlf and Slice, 1990). As shown in previous studies, GPA performed on landmarks taken at both the proximal and distal ends of a long bone will emphasize variation related to long bone length and joint size (e.g., Tallman, 2012; Rein et al., 2015). Therefore, regression analyses (described below) were performed to identify additional aspects of variation, beyond bone length and joint size, that distinguished taxa in our sample.

We performed principal component analyses (PCA) on the resulting Procrustes coordinates in MorphoJ 1.05a (Klingenberg, 2011). This allowed us to examine clustering patterns among extant and extinct taxa without providing a priori information about phylogenetic relationships or functional similarities. Thus, clustering of species is solely the result of measured skeletal shape variation, and these similarities may reflect similar locomotor repertoires. Separate principal component analyses were performed on the 17-landmark coordinate configurations for all individuals and for the means of the extant species and the two fossil hominins to examine adaptations to FSL in the complete ulna of these species of Australopithecus. All PCA scatter plots were made in PAST (Hammer et al., 2001). 
A PCA of individual landmark configurations was performed to explore clustering patterns among individuals of each extant and extinct species. However, since published FSL proportion data on extant primates used in this study were species averages, a PCA was run on mean landmark configurations of extant species in order to regress principal component (PC) scores against logit-transformed mean species FSL proportion data, and a size variable using multiple regression and a phylogenetic generalized least-squares (pGLS) model based on Pagel's $\lambda$ transformation using a restricted maximum likelihood estimate of Pagel's $\lambda$ (Pagel, 1999; Freckleton et al., 2002; Spoor et al., 2007). This modeling procedure estimates the degree of phylogenetic signal in the data and computes regression parameters based on this estimate, allowing us to take into account phylogeny while examining the relationship between aspects of shape variation represented along PCs 1 and 2 and FSL. Proportion values of zero were transformed following the procedure described by Warton and Hui (2011) in order to linearize the data so that they could be used in regression analysis. Proportion values between 0.01 and 0.99 that are logit-transformed (using the natural logarithm) will fall between -4.6 and 4.6, with proportion values of 0.50 transforming to 0 . Since proportion values equal to zero transform to an undefined value $(-\infty)$, we followed the protocol described by Warton and Hui (2011) of adding to the numerator and denominator of the logit function the smallest non-zero value in our proportion data (0.01) to allow the inclusion of taxa with proportion values of zero. Regression analyses were performed in R (R Core Team, 2012) using the packages 'ape' (Paradis et al., 2004) and 'nlme' (Pinheiro et al., 2012). The inferred phylogenetic tree used in this analysis was downloaded from the 10kTrees project website (Arnold et al., 2010). We used two different size variables. First, we employed log-transformed centroid size (CS), which is a commonly used size variable in geometric morphometrics that characterizes the overall size of the object being 
measured. We also employed a ln-transformed ulnar size surrogate (Ulna SS) described by Drapeau (2004). This size surrogate is based on the geometric mean of five interlandmark distance measurements of the ulna, including the proximodistal length of the trochlear notch, maximum depth of the trochlear notch, proximodistal length of the radial notch, the anteroposterior diameter of the coronoid process, and the anteroposterior length of the midshaft (Drapeau, 2004).

We tested whether or not our shape analysis was driven only by ulnar length and size by employing the same mean extant species PC scores described in the previous paragraph and by performing a series of multiple linear regressions in which we regressed either PC score 1 or 2 against three independent variables: ulnar length, ulnar joint size, and a ratio representing the shape of either the trochlear notch or the radial notch of the proximal ulna. The shape of the trochlear notch was represented by a ratio of the proximodistal length of the trochlear notch and its maximum mediolateral breadth. The shape of the radial notch was represented by the ratio of the anteroposterior to the proximodistal lengths of the articular surface. These variables were chosen based on visual examination of the warped ulnar surfaces along PCs 1 and 2 published in Rein et al. (2015). We also performed two additional multiple linear regression analyses. First, we regressed the logit-transformed FSL proportion data against mean species PC scores (representing PCs 1 and 2). Next, we regressed the same logit-transformed proportion data against mean species ln-transformed ulnar length and the ln-transformed ulnar size surrogate. These analyses were performed to compare the strength of the relationship between FSL and the shape variables resulting from the GPA versus that between FSL and traditional linear measurements of ulnar length and joint size. 
Similar to the method described by Rein et al. (2015), we employed principal component regression on mean species Procrustes coordinate data to estimate the degree to which the ulnae of A. sediba and A. afarensis were adapted to FSL. This analysis was performed using the 'pls' package (Mevik and Wehrens, 2007) in R (R Core Team, 2012). Since the regression model is based on mean species data, but the fossils are individual representatives of extinct hominin species, we examined the range of estimates for all individual specimens (i.e., same number of specimens as indicated for each taxon in Table 1) of each extant species to assess the degree of overlap among individual estimates of different extant species, and further to understand how reliable individual fossil estimates were. In this analysis, each individual specimen from an extant species was analyzed as if it was a lone fossil specimen. These estimates were then compared to estimates for the fossil hominins.

Procrustes distances (Rohlf, 2000) were also calculated between each hominin landmark configuration and the mean landmark configurations of each extant species in MorphoJ (Klingenberg et al., 2011). Visualization of shape differences between the fossil ulnae and mean landmark coordinate configurations of extant hominids was performed in the EVAN toolbox (available through the EVAN-Society, www.evan-society.org) using a Thin Plate Spline algorithm to warp a surface scan of a human ulna. This type of visualization analysis is useful in identifying aspects of shape that distinguish the bones of different species.

\section{Results}

Results of the PCA that included extant and extinct species are presented in Figure 2 and in Tables 3 and 4 . Although the A. sediba ulna fell within the $95 \%$ confidence ellipse for chimpanzees, it was characterized by a lower PC 1 score than those of all measured specimens 
representing chimpanzees, humans, and gorillas (Fig. 2a). It was located outside of the Homo sapiens cluster and closer to those of Pan troglodytes and Pongo pygmaeus, two species that perform low proportions of FSL when moving in an arboreal setting (Cant, 1987; Hunt, 1991; Thorpe and Crompton, 2006). U.W. 88-62 was characterized by a relatively low PC 1 score comparable to that of suspensory orangutans (Fig. 2a). In the mean shape analysis, the PC 2 score for U.W. 88-62 was lower than those of average species landmark coordinate configurations of extant hominines (i.e., African apes and humans) and higher than that of Pongo (Fig. 2b). Australopithecus afarensis, on the other hand, fell within the chimpanzee cluster, but in the area of overlap between human and chimpanzee ranges of variation (Fig. 2a). The A.L. 438-1 specimen was characterized by higher PC 1 and 2 scores than those of the A. sediba ulna. We examined the relationship between each set of PC scores and proportions of FSL when incorporating information on size and phylogeny using only mean extant species data. Both PC 1 and PC 2 had a significant relationship with FSL proportion when size was taken into account (Table 5). These relationships were significant regardless of which size variable was employed (i.e., log-transformed centroid size or the ln-transformed ulnar size surrogate based on the geometric mean of five measurements).

Based on estimates of Pagel's $\lambda$ (Table 5), regression equations for PC 1 were estimated to have higher degrees of phylogenetic signal than those of PC 2 . The higher phylogenetic signal characterizing PC 1 can be explained by the absence of overlap between hominid mean PC 1 scores and those of more distantly related taxa. Furthermore, all three atelid taxa were found to have relatively low PC 1 scores. In contrast, PC 2 scores exhibited greater overlap among distantly related taxa. The PC 2 score for the mean Ateles landmark configuration, for example, 
was quite low and fell between those of Pongo and Gorilla, whereas those of other atelids were much higher.

When controlling for log-transformed centroid size, extant species that have a FSL frequency comprising a greater proportion of the overall locomotor repertoire than 0.01 were characterized by lower mean PC 1 scores than closely related taxa that perform less suspension (Fig. 3a). When similarly controlling for size, scores along PC 2 were lower for species that perform relatively high frequencies of FSL compared to other taxa (whether closely or distantly related, Fig. 3b). Australopithecus sediba and A. afarensis had lower PC 1 scores than that of the mean score for $H$. sapiens, a closely related species with a similar centroid size. Unlike A. afarensis, however, A. sediba also scored lower on PCs 1 and 2 than P. troglodytes (Fig. 2b).

Positions of the fossil ulnae on the PCA scatter plots (Fig. 2) indicated that, when making comparisons to average hominine shape configurations, the U.W. 88-62 A. sediba ulna was relatively long (compared to joint size) with more equal proximodistal and mediolateral lengths (squarish appearance as opposed to rectangular; Fig. 4). This shape difference in the trochlear notch of $A$. sediba compared to that of $A$. afarensis is evident in Figure 5. Additionally, the overall smaller appearance of the A. sediba trochlear notch versus that of A. afarensis following Procrustes superimposition (see Methods section) resulted from relatively greater length of the $A$. sediba ulna compared to that of $A$. afarensis. There were also differences in radial notch shape along PCs 1 and 2. Lower PC scores for both axes corresponded to more circular radial notches.

In regression analysis, scores along both PCs 1 and 2 had a significant relationship with ulnar length and joint size (Table 6). However, bone length and joint size were not the only variables to be characterized by a significant relationship with PC 2 scores. Shapes of the trochlear notch and radial notch significantly corresponded with PC 2 scores when ulnar length and size were 
taken into account (Table 6). Mean species data points with lower PC 2 scores were represented by not only a relatively longer ulna but also a trochlear notch that was more squarish than rectangular, and a radial notch that was slightly more circular. Regarding the strength of the relationships among FSL and measurements of ulnar shape and size, Table 7 presents R-squared values for multiple regression analyses in which FSL is the dependent variable. A stronger relationship and lower residual standard error were found between FSL and PC scores from the GPA compared to those reported for the regression involving FSL and measurements of ulnar length and size (i.e., Ulna SS).

Estimates of FSL based on PC regression are presented in Figure 6. Only PCs 1 and 2 were included, based on cross-validation analysis and visual inspection of clustering patterns using additional principal components. On average, semi-terrestrial taxa were characterized by higher FSL estimates than those of the non-suspensory arboreal quadrupeds, such as Alouatta, and the terrestrial biped, Homo. With regard to the fossils, the estimate of FSL for A. sediba only overlapped with taxa that perform FSL (i.e., Pan, Gorilla, Presbytis, and Lagothrix) and was outside the range of non-suspensory taxa. In contrast, the estimate of FSL for A. afarensis overlapped with both non-suspensory taxa and those that perform low proportions of FSL.

Analysis of overall ulnar shape confirms the results of the PCAs. Procrustes distances between mean shape configurations of hominids (great apes, Australopithecus, and Homo sapiens) are presented in Table 8. Landmark coordinate configurations of both fossil ulnae shared the smallest distance with the mean configuration of Pan. The next smallest distances involving A. sediba were with $A$. afarensis and Pongo. In contrast, A. afarensis exhibited the next smallest distance with Homo rather than with Pongo. Thus, while the ulnae of both $A$. sediba and A. afarensis were most similar to Pan, A. sediba was characterized by the next 
greatest similarity to Pongo, whereas A. afarensis was next most similar to Homo. This implies a stronger FSL signal in the overall shape of the A. sediba ulna than that of A. afarensis.

\section{Discussion}

The limb skeletons of Australopithecus species are characterized by unique combinations of primitive and derived hominin features (e.g., Stern and Susman, 1983; Ricklan, 1987; Kivell et al., 2011). The question remains, however, as to whether these suites of morphological features indicate adaptation to arboreal locomotor behaviors, such as suspension, or are simply retained as vestiges of the evolutionary past due to disuse or selective neutrality. One challenge to answering this question involves identifying those areas of the skeleton that are most informative about adaptation to locomotion. Results of the present study demonstrate that $A$. sediba, more so than A. afarensis, exhibits similarities in ulnar articular surface configurations with several living primates that have non-trivial FSL components to their locomotor repertoires.

We focused on a particular set of arboreal locomotor behaviors in which the forelimb plays a major role. Given the biomechanical demands placed on the forelimb skeleton during brachiation and forelimb swing, we chose to examine forelimb-dominated suspensory movements since they likely impose a strong selective pressure on musculoskeletal structure of the antebrachium. Previous research has demonstrated that variation in ulnar shape, including relative ulnar length, corresponds to variation in performance of forelimb suspensory locomotion in anthropoid primates (Drapeau and Ward, 2007; Rein et al., 2015).

Our results showed that the first two axes of our PCA, when accounting for size and phylogeny, corresponded to the degree of FSL performed by each anthropoid species in our sample. The distinction between the two hominin fossils is due primarily to the relatively 
smaller proximal joint size of the A. sediba U.W. 88-62 ulna (characterized by a relatively short proximodistal length) observed after GPA. Procrustes superimposition scales each specimen to a common centroid size. Centroid size of the landmark configurations examined in this study was largely based on ulnar length. Thus, each shape configuration resulting from the generalized Procrustes analysis looked similar in ulnar length, but varied in terms of joint size. As reported by Rein et al. (2015), small joint sizes relative to centroid size characterize extant hominoids, platyrrhines, and cercopithecoids that perform substantial proportions of forelimb suspensory locomotion. While both fossils are closest to the chimpanzee mean configuration, the A. sediba specimen was found to be more similar to the mean configuration observed in the extant hominid that performs the most FSL (i.e., Pongo) than the A. afarensis fossil. Although A.L. 438-1 clustered with individuals of Pan along PCs 1 and 2, the interpretation of its functional significance is more ambiguous than that of U.W. 88-62 since it is located in an area of overlap between two groups (i.e., chimpanzees and humans). The difference between $A$. sediba and $A$. afarensis is also observed in estimates of FSL based on PC regression, in which the estimate for A. sediba only overlapped with those of species characterized by low proportions of suspension, whereas that of A. afarensis was even lower and also overlapped with non-suspensory species.

Drapeau and Ward (2007) reported that relative ulnar length reflects locomotor adaptation among hominoids. They found that highly suspensory Asian apes were characterized by the longest ulnae, followed by Pan, Gorilla, and humans (the latter with the shortest ulnae). The clustering patterns of humans, gorillas, and chimpanzees along PCs 1 and 2 relate, in part, to these differences in relative ulnar length (Table 6). Thus, the position of the U.W. 88-62 specimen on the PCA scatter plot partially relates to it being long relative to joint size. However, as described by Rein et al. (2015), variation in comprehensive ulnar shape configurations 
corresponds to other joint shape differences captured by our landmark dataset as well. In this study, PC 2 scores are also related to variation in the shapes of the trochlear and radial notches (Table 6). Taxa that perform FSL were characterized by, on average, trochlear notches that were more square-shaped than rectangular (i.e., appearing relatively short proximodistally and wider mediolaterally compared to those of other taxa) and radial notches that were more circular (relatively shorter anteroposteriorly and wider proximodistally compared to those of other taxa). While the U.W. 88-62 fossil fell between the averages of Pan and Pongo along PC 2, A.L. 438-1 was located between those of humans and Pan. These results support previous findings that the A.L. 438-1 ulna is characterized by more human-like features than later hominin ulnae (McHenry et al., 2007).

The relative length of the ulna could be related to the demands of forelimb suspensory locomotion. Drapeau (2004) reported that suspensory species are characterized by elongated ulnae. Elongated forearms would enhance the reach of suspensory anthropoid primates as they move along the substrate. Rein et al. (2015) and the present study have built upon this research by employing geometric morphometrics to combine relative ulnar length and other aspects of ulnar shape (discussed below) that correspond to suspensory locomotion across anthropoid primates and construct a predictive model to examine the role of forelimb suspensory locomotion in the repertoire of extinct anthropoids (including hominins).

The trochlear notch is part of the humeroulnar joint that is concerned with flexion and extension at the elbow joint. Previous examinations of trochlear notch shape across different anthropoid clades using traditional ratios have found overlap among hominoids and other taxa (e.g., Larson, 1998). As described by Rose (1988), the humeroulnar joint should provide stability during all flexion/extension positions. If so, the hard and soft tissue anatomy should be 
adapted to buttress the joint against mediolateral forces that might result in dislocations during forelimb suspensory locomotion. In the present study, there was a relationship between relatively greater mediolateral breadth of the trochlear notch and performance of FSL, and increasing the mediolateral width of the joint surface of the proximal ulna decreases the size of the force required to balance rotational forces along the long axis of the bone in suspensory locomotion (Sarmiento (1985). The proximal radioulnar joint is involved in pronation/supination movements of the forearm (Rose, 1998). The more circular shape of the radial notch characterizing suspensory species reported in the present study might relate to the need for stability at this joint throughout the extensive range of pronation/supination that occurs at various levels of elbow flexion/extension associated with suspensory activities (Rose, 1998).

Since smaller $A$. afarensis fossils could not be included in the present analysis (given the lack of complete specimens) and a complete ulna for a large-bodied $A$. sediba individual does not exist, there remains the possibility that the distinctions observed between the smaller $A$. sediba ulna and the larger A. afarensis specimen are based on differences in the size of the bone or sexual dimorphism. However, Drapeau et al. (2005) were able to compare the forelimb skeletons (including the ulnae) of large (A.L. 438-1) and small (A.L. 288-1) individuals of $A$. afarensis. They reported that these two individuals had matching forelimb features in almost all cases and did not differ in functional anatomy. Thus, while the potential effects of allometry cannot be discounted from the present comparison of two different extinct hominin species, previous research suggests that allometry does not play a major role in intraspecific shape variation of the ulna, at least for A. afarensis.

The bones of the arm and forearm of $A$. sediba have been described as primitive for hominins, suggesting that this species was adapted to suspensory and climbing behaviors (Churchill et al., 
2013). The results of our geometric morphometric analysis of complete ulnar shape support this suggestion, and we propose that this species was adapted to perform forelimb suspensory locomotion only in low frequencies, similar to P. troglodytes or Presbytis melalophos. The U.W. 88-62 specimen remained adapted for these behaviors despite modifications to the hand that suggest the selective importance of more human-like uses of the upper limb (Kivell et al., 2011). Moreover, the combination of character states in the limb skeleton of A. sediba has been described as distinct from that of earlier Australopithecus, suggesting a distinct locomotor behavior for this taxon. While our results do not unequivocally demonstrate a different locomotor adaptation in A. sediba compared to A. afarensis, they do indicate a stronger signal for suspensory behaviors in the former.

Australopithecus sediba is not the only early hominin from South Africa to be characterized as more adapted to arboreal locomotor behaviors than A. afarensis. Australopithecus africanus has also been reported to have more ape-like limb proportions than A. afarensis (McHenry and Berger, 1998; Green et al., 2007). An ulna from A. africanus was not included in the present study since there was no specimen on which comparable measurements could be taken. Given the current consensus regarding phylogenetic relationships between Australopithecus species, the differences between A. afarensis and A. africanus were described as being either the result of a secondarily-derived ape-like condition in A. africanus, or independently evolved limb proportions in Homo and A. afarensis (Green et al., 2007). In either scenario, Green et al. (2007) argued that this was evidence for each species of Australopithecus being under different selection pressures related to arboreal locomotion.

Whether the importance of arboreal movements such as FSL led to secondarily derived features in the A. sediba ulna or perhaps promoted the primitive retention of these traits is not 
clear. However, the more human-like shape of the complete ulna of A. afarensis and the inferred phylogenetic relationships among species of Australopithecus and Homo (Irish et al., 2013; Dembo et al., 2015) suggest that $A$. sediba was under different selection pressures than $A$. afarensis, leading to a divergent postcranial skeletal morphology in these hominin species. For example, there are a number of derived bipedal features in the A. sediba pelvis that do not characterize earlier australopiths (Kibii et al., 2011). One example is the orientation of the iliac blade, which would have provided a more human-like hip abductor mechanism. Examination of the hind limb anatomy of $A$. sediba has led to the inference that this species may have performed a different form of bipedalism compared to other australopiths (DeSilva et al., 2013). Given the adaptations found in the pelvis, selective pressures during the approximately one million years following the disappearance of A. afarensis may have resulted in a more efficient form of bipedalism characterizing A. sediba. However, these derived features related to bipedalism were found in combination with hindlimb traits associated with arboreal locomotion (e.g., Zipfel et al., 2011), indicating that the use of an arboreal environment also retained importance for A. sediba. The results of the current study suggest that below branch activities may have been one of those arboreal behaviors, and it may have played a larger role in the locomotor repertoire of A. sediba than in that of A. afarensis. If that were the case, it would explain the combination of derived hindlimb features related to bipedalism and forearm features related to suspensory locomotion. In conclusion, our examination of a wide range of extant anthropoid primate ulnae suggests that forelimb suspensory locomotion may have played a small but adaptively significant role in the arboreal positional repertoire of A. sediba.

\section{Acknowledgments}


Thanks to the Evolutionary Studies Institute, the University of Witwatersrand, Lee Berger, Steven Churchill, Bernhard Zipfel, and the Fossil Access Committee for providing access to the Australopithecus sediba fossil. Thanks to Authority for Research and Conservation of Cultural Heritage, the National Museum of Ethiopia, Mamitu Yilma, and William Kimbel for providing access to the Australopithecus afarensis specimen. Extant species museum specimens were generously made available for study by Linda Gordon (National Museum of Natural History), Judith Chupasko (Harvard Museum of Comparative Zoology), William Stanley (Field Museum of Natural History), Malcolm Harman (Powell-Cotton Museum), Paula Jenkins and Louise Tomsett (Natural History Museum, London), Frieder Mayer (Humboldt-Universität zu Berlin, Museum für Naturkunde), Emmanuel Gilissen and Wim Wendelen (Royal Museum for Central Africa), Marcia Ponce de León and Christoph Zollikofer (Anthropologisches Institut und Museum, Universität Zürich-Irchel), Emma Mbua and Fredrick Kyalo Manthi (National Museums of Kenya), Yohannes Haile-Selassie and Lyman Jellema (Hamann-Todd Collection),

Nancy Simmons, Neil Duncan, and Eileen Westwig (American Museum of Natural History), and Michael Francken (University of Tübingen). The research was funded by the Volkswagen Foundation (Germany; TRR), National Science Foundation (Grant numbers BCS-0849204, IGERT 0333415; USA; TRR and TH), L.S.B. Leakey Foundation (USA; TRR), Wenner-Gren Foundation (USA; TRR), and New York University (USA; TRR).

\section{References}

Arnold, C., Matthews, L.J., Nunn., C.L., 2010. The 10kTrees Website: A new online resource for primate phylogeny. Evol. Anthropol. 19, 114-118.

Berger, L.R., de Ruiter, D.J., Churchill, S.E., Schmid, P., Carlson, K.J., Dirks, P.H.G.M., Kibii, J.M., 2010. Australopithecus sediba: A new species of Homo-like australopith from 
South Africa. Science 328, 195-204.

Cant, J.G.H., 1987. Positional behavior of female Bornean orangutans (Pongo pygmaeus). Am. J. Primatol. 12, 71-90.

Chiu C.-H., Hamrick, M.W., 2002. Evolution and the development of the primate limb skeleton. Evol. Anthropol. 11, 94-107.

Churchill, S.E., Holliday, T.W., Carlson, K.J., Jashashvili, T., Macias, M.E., Mathews, S., Sparling, T.L., Schmid, P., de Ruiter, D.J., Berger, L.R., 2013. The upper limb of Australopithecus sediba. Science 340, 1233477-1-1233477-6.

Cooke, C.A., 2012. The feeding, ranging, and positional behaviors of Cercocebus torquatus, the red-capped mangabey, in Sette Cama Gabon: A phylogenetic perspective. Ph.D. Dissertation, The Ohio State University.

Defler, T.R., 1999. Locomotion and posture in Lagothrix lagotricha. Folia Primatol. 70, 313327.

Dembo, M., Matzke, N.J., Mooers, A.Ø., Collard, M., 2015. Bayesian analysis of a morphological supermatrix sheds light on controversial fossil hominin relationships. Proc. R. Soc. B 282, 20150943.

DeSilva, J.M., Holt, K.G., Churchill, S.E., Carlson, K.J., Walker, C.S., Zipfel, B., Berger, L.R., 2013. The lower limb and mechanics of walking in Australopithecus sediba. Science 340, 1232999-1-1232999-5.

Dirks, P.H.G.M., Kibii, J.M., Kuhn, B.F., Steininger, C., Churchill, S.E., Kramers, J.D., Pickering, R., Farber, D.L., Mériaux, A-S, Herries, A.I.R., King, G.C.P., Berger, L.R., 2010. Geological setting and age of Australopithecus sediba from southern Africa. Science 328, 205-208. 
Drapeau, M.S.M., 2004. Functional anatomy of the olecranon process in hominoids and PlioPleistocene hominins. Am. J. Phys. Anthropol. 124, 297-314.

Drapeau, M.S.M., 2008. Articular morphology of the proximal ulna in extant and fossil hominoids and hominins. J. Hum. Evol. 55, 86-102.

Drapeau, M.S.M., Ward, C.V., 2007 Forelimb segment length proportions in extant hominoids and Australopithecus afarensis. Am. J. Phys. Anthropol. 132, 327-343.

Drapeau, M.S.M., Ward, C.V., Kimbel, W.H., Johanson, D.C., Rak, Y., 2005. Associated cranial and forelimb remains attributed to Australopithecus afarensis from Hadar, Ethiopia. J. Hum. Evol. 48, 593-642.

Duncan, A.S., Kappelman, J., Shapiro, L.J., 1994. Metatarsophalangeal joint function and positional behavior in Australopithecus afarensis. Am. J. Phys. Anthropol. 93, 67-81.

Fleagle, J.G., 1980. Locomotion and posture. In: Chivers, D.J. (Ed.), Malayan Forest Primates: Ten Years' Study in Tropical Rain Forests. Plenum Press, New York, pp. 191-207.

Fleagle, J.G., 2013. Primate Adaptation and Evolution, $3^{\text {rd }}$ ed. Academic Press, San Diego.

Freckleton, R.P., Harvey, P.H., Pagel, M.D., 2002. Phylogenetic analysis and comparative data: a test and review of evidence. Am. Nat. 160, 712-726.

Gebo, D.L., 1996. Climbing, brachiation, and terrestrial quadrupedalism: historical precursors of hominid bipedalism. Am. J. Phys. Anthropol. 101, 55-92.

Gould, S.J., Lewontin, R.C., 1979. The spandrels of San Marco and the Panglossian paradigm: a critique of the adaptationist programme. Proc. R. Soc. Lond. B. Biol. Sci. 205, 581-598.

Gould, S.J., Vrba, E.S., 1982. Exaptation-a missing term in the science of form. Paleobiology 8, $4-15$.

Gower, J.C., 1975. Generalized Procrustes analysis. Psychometrika 40, 33-51. 
Green, D.J., Alemseged, Z., 2012. Australopithecus afarensis scapular ontogeny, function, and the role of climbing in human evolution. Science 338, 514-517.

Green, D.J., Gordon, A.D., Richmond, B.G., 2007. Limb-size proportions in Australopithecus afarensis and Australopithecus africanus. J. Hum. Evol. 52, 187-200.

Hallgrímsson, B., Willmore, K., Hall, B.K., 2002. Canalization, developmental stability, and morphological integration in primate limbs. Yearb. Phys. Anthropol. 45, 131-158.

Hammer, Ø., Harper, D.A.T., Ryan, P.D., 2001. PAST: paleontological statistics software package for education and data analysis. Palaeontol. Electron. 4, http://palaeoelectronica.org/2001_1/past/issue1_01.htm.

Hunt, K.D., 1991. Positional behavior in the Hominoidea. Int. J. Primatol. 12, 95-118.

Irish, J.D., Guatelli-Steinberg, D., Legge, S.S., de Ruiter, D.J., Berger, L.R., 2013. Dental morphology and the phylogenetic "place" of Australopithecus sediba. Science 340, 1233062.

Jungers, W.L., 1982. Lucy’s limbs: skeletal allometry and locomotion in Australopithecus afarensis. Nature 297, 676-678.

Kibii, J.M., Churchill, S.E., Schmid, P., Carlson, K.J., Reed, N.D., de Ruiter, D.J., Berger, L.R., 2011. A partial pelvis of Australopithecus sediba. Science 333, 1407-1411.

Kivell, T.L., Kibii, J.M., Churchill, S.E., Schmid, P., Berger, L.R., 2011. Australopithecus sediba hand demonstrates mosaic evolution of locomotor and manipulative abilities. Science 333, $1411-1417$.

Klingenberg, C.P., 2011. MorphoJ: an integrated software package for geometric morphometrics. Mol. Ecol. Res. 11, 353-357.

Larson, S.G., 1998. Parallel evolution in the hominoid trunk and forelimb. Evol. Anthropol. 6, 
87-99.

Latimer, B., 1991. Locomotor adaptations in Australopithecus afarensis: the issue of arboreality. In: Senut, B., Coppens, Y. (Eds.), Origine(s) de la bipédie chez les Hominidés. CNRS, Paris, pp. 169-176.

Latimer, B., Lovejoy, C.O., 1990a. Hallucal tarsometatarsal joint in Australopithecus afarensis.

Am. J. Phys. Anthropol. 82, 125-133.

Latimer, B., Lovejoy, C.O., 1990b. Metatarsophalangeal joints of Australopithecus afarensis. Am. J. Phys. Anthropol. 83, 13-23.

Lovejoy, C.O., Cohn, M.J., White, T.D., 1999. Morphological analysis of the mammalian postcranium: A developmental perspective. Proc. Natl. Acad. Sci. USA 96, 1324713252.

McHenry, H.M., Berger, L.R., 1998. Body proportions in Australopithecus afarensis and A. africanus and the origin of the genus Homo. J. Hum. Evol. 35, 1-22.

McHenry, H.M., Brown, C.C., McHenry, L.J., 2007. Fossil hominin ulnae and the forelimb of Paranthropus. Am. J. Phys. Anthropol. 134, 209-218.

Mevik, B.H., Wehrens, R., 2007. The pls package: Principal component and partial least-squares regression in R. J. Stat. Softw. 18, 1-23.

Ohman, J.C., Krochta, T.J., Lovejoy, C.O., Mensforth, R.P., Latimer, B., 1997. Cortical bone distribution in the femoral neck of hominoids: implications for the locomotion of Australopithecus afarensis. Am. J. Phys. Anthropol. 104, 117-131.

Pagel, M., 1999. Inferring the historical patterns of biological evolution. Nature 401, 877-884.

Paradis, E., Claude, J., Strimmer, K., 2004. APE: analyses of phylogenetics and evolution in R language. Bioinformatics 20, 289-290. 
Pickering, R., Dirks, P.H.G.M, Jinnah, Z., de Ruiter, D.J., Churchill, S.E., Herries, A.I.R, Woodhead, J.D., Hellstrom, J.C., Berger, L.R., 2011. Australopithecus sediba at 1.977 Ma and implications for the origins of the genus Homo. Science 333, 1421-1423.

Pinheiro, J., Bates, D., DebRoy, S., Sarkar, D., R Development Core Team, 2012. nlme: Linear and nonlinear mixed effects models. R package version 3.1-105.

Prang, T.C., 2015. Calcaneal robusticity in Plio-Pleistocene hominins: Implications for locomotor diversity and phylogeny. J. Hum. Evol. 80, 135-146.

R Core Team, 2012. R: A language and environment for statistical computing. $\mathrm{R}$ Foundation for Statistical Computing: Vienna, Austria. http://www.R-project.org/.

Rein, T.R., Harvati, K., Harrison, T., 2015. Inferring the use of forelimb suspensory locomotion by extinct primate species via shape exploration of the ulna. J. Hum. Evol. 78, 70-79.

Ricklan, D.E., 1987. Functional anatomy of the hand of Australopithecus africanus. J. Hum. Evol. 16, 643-664.

Rohlf, F.J., 2000. Statistical power comparisons among alternative morphometric methods. Am. J. Phys. Anthropol. 111, 463-478.

Rohlf, F.J., Slice, D., 1990. Extensions of the Procrustes method for the optimal superimposition of landmarks. Syst. Zool. 39, 40-59.

Rose, M.D., 1988. Another look at the anthropoid elbow. J. Hum. Evol. 17, 193-224.

Sarmiento, E.E., 1985. Functional Differences in the Skeleton of Wild and Captive Orangutans and Their Adaptive Significance. Ph.D. Dissertation, New York University.

Senut, B., 1980. New data on the humerus and its joints in Plio-Pleistocene hominids. Coll. Anthropol. 1, 87-93.

Shubin, N., Tabin, C., Carroll, S., 1997. Fossils, genes and the evolution of animal limbs. Nature 
$388,639-648$.

Spoor, F., Garland, T. Jr., Krovitz, J., Ryan, T.M., Silcox, M.T., Walker, A., 2007. The primate semicircular canal system and locomotion. Proc. Natl. Acad. Sci. U.S.A. 104, 1080810812.

Stern, J.T. Jr., 2000. Climbing to the top: a personal memoir of Australopithecus afarensis. Evol. Anthropol. 9, 113-133.

Stern, J.T. Jr., Susman, R.L., 1983. The locomotor anatomy of Australopithecus afarensis. Am. J. Phys. Anthropol. 60, 279-317.

Susman, R.L., Stern Jr., J.T., Jungers, W.L., 1984. Arboreality and bipedality in the Hadar hominids. Folia Primatol. 43, 113-156.

Tallman, M., 2012. Morphology of the distal radius in extant hominoids and fossil hominins: Implications for the evolution of bipedalism. Anat. Rec. 295, 454-464.

Tallman, M., 2013. Forelimb to hindlimb shape covariance in extant hominoids and fossil hominins. Anat. Rec. 296, 290-304.

Thorpe, S.K.S., Crompton, R.H., 2006. Orangutan positional behavior and the nature of arboreal locomotion in Hominoidea. Am. J. Phys. Anthropol. 131, 384-401.

Ward, C.V., 2002. Early hominin posture and locomotion: where do we stand? Yearb. Phys. Anthropol. 45, 185-215.

Warton, D.I., Hui, F.K.C., 2011. The arcsine is asinine: the analysis of proportions in ecology. Ecology 92, 3-10.

Youlatos, D., 1998. Positional behavior of two sympatric Guianan capuchin monkeys, the brown capuchin (Cebus apella) and the wedge-capped capuchin (Cebus olivaceus). Mammalia 62, 351-365. 
Zipfel, B., DeSilva, J.M., Kidd, R.S., Carlson, K.J., Churchill, S.E., Berger, L.R., 2011. The foot and ankle of Australopithecus sediba. Science 333, 1417-1420. 


\section{Figure legends}

Figure 1. Rendering of reconstructed U.W. 88-62. Views: a) anterior, b) medial, c) posterior, d) lateral, e) proximal, f) distal. Scale bar $=40 \mathrm{~mm}$.

Figure 2. a) Scatter plot with $95 \%$ confidence ellipses of the first two principal component (PC) scores of shape variation characterizing 17-landmark coordinate configurations. Principal components 1 and 2 account for $44.5 \%$ and $18.0 \%$ of the overall variance, respectively. b) Scatter plot of mean species shape configurations. Principal components 1 and 2 account for $53.8 \%$ and $20.8 \%$ of the overall variance, respectively.

Figure 3. Scatter plots of mean species principal component (PC) scores against log-transformed centroid size (CS) showing relationship between PC scores and performance of forelimb suspensory locomotion when size is taken into account. a) PC 1 scores, b) PC 2 scores.

Figure 4. Shape changes for principal component (PC) axes 1 and 2 visualized through warping of a human ulnar surface scan in anterior (top) and lateral (bottom) view. Highlighted regions represent areas of the bone where landmarks were collected.

Figure 5. Surface reconstruction of human ulna warped to mean species shape of 17-landmark configurations for hominids in anterior (top) and lateral (bottom) view; a) Gorilla gorilla, b) Homo sapiens, c) Australopithecus afarensis, d) Pan troglodytes, e) Australopithecus sediba, f) Pongo pygmaeus. Highlighted regions represent areas of the bone where landmarks were collected.

Figure 6. Plot of individual estimates of forelimb suspensory locomotion (FSL) proportions. Red squares $=$ arboreal quadrupeds, blue squares $=$ semi-terrestrial quadrupeds, pink squares $=$ species that perform FSL (ordered in increasing proportion of FSL). 
Table 1. Species included in the analysis and their proportion of forelimb suspensory locomotion

\begin{tabular}{|c|c|c|}
\hline Species & $n$ & Mean FSL ${ }^{\mathrm{a}}$ \\
\hline Alouatta seniculus & 20 & 0.00 \\
\hline Ateles geoffroyi & 16 & 0.25 \\
\hline Cebus apella & 20 & 0.00 \\
\hline Cercocebus torquatus & 10 & 0.00 \\
\hline Cercopithecus ascanius & 9 & 0.00 \\
\hline Cercopithecus mitis & 20 & 0.00 \\
\hline Chlorocebus aethiops & 20 & 0.00 \\
\hline Gorilla gorilla & 20 & 0.01 \\
\hline Homo sapiens $^{\mathrm{b}}$ & 26 & 0.00 \\
\hline Hylobates lar & 20 & 0.50 \\
\hline Lagothrix lagotricha & 18 & 0.07 \\
\hline Lophocebus albigena & 16 & 0.00 \\
\hline Macaca fascicularis & 20 & 0.00 \\
\hline Macaca nemestrina & 15 & 0.00 \\
\hline Pan troglodytes & 22 & 0.01 \\
\hline Papio anubis & 20 & 0.00 \\
\hline Pongo pygmaeus & 20 & 0.10 \\
\hline Presbytis melalophos & 15 & 0.03 \\
\hline Trachypithecus obscurus & 20 & 0.00 \\
\hline Australopithecus afarensis ${ }^{\mathrm{c}}$ & 1 & - \\
\hline Australopithecus sediba ${ }^{\mathrm{d}}$ & 1 & - \\
\hline
\end{tabular}


${ }^{a}$ Proportion of total locomotor behavior (arboreal and terrestrial) dedicated to forelimb suspensory locomotion (FSL). These data are taken from Rein et al. (2015) who referenced Fleagle (1980, 2013), Cant (1987), Gebo (1996), Youlatos (1998), Defler (1999), and Cooke (2012) as sources for the data they employed.

${ }^{\mathrm{b}}$ Human sample includes ulnae from 10 African Americans (Hamann-Todd Collection), 10 European Americans (Hamann-Todd Collection), four individuals from a medieval German population (Paleoanthropology osteological collections, University of Tübingen), one individual from a Maasai population (Paleoanthropology osteological collections, University of Tübingen), and one individual from a Tunisian population (Paleoanthropology osteological collections, University of Tübingen).

${ }^{\mathrm{c}}$ Australopithecus afarensis is represented by A.L. 438-1a, a left ulna.

${ }^{\mathrm{d}}$ Australopithecus sediba is represented by U.W. 88-62, a right ulna. 
Table 2. Anatomical locations of landmarks collected on the proximal and distal ulna. ${ }^{\text {a }}$

\section{Description}

1

2

3

4

5

6

7

8

9

10

11

12

13

14

15

16

17
Most proximal point on medial margin of trochlear facet

Most proximal point on lateral margin of trochlear facet

Location of maximum constriction on medial margin of trochlear notch

Deepest point along the midline of trochlear facet

Location of maximum constriction on lateral margin of trochlear notch

Most medial point on distal border of trochlear notch

Most anterior point on coronoid process

Point of intersection of radial and trochlear facets

Most posterior point on radial facet

Most anterior point of radial facet

Point of maximum depth in radial facet

Ulnar head medialmost point

Ulnar head anteriormost point

Ulnar head lateralmost point

Ulnar head distalmost point (constrained to midline)

Styloid process distalmost point

Posteriormost point on distal ulna

\footnotetext{
${ }^{\mathrm{a}}$ Landmarks used in this study were based on several published studies including Drapeau
} (2008), Tallman (2013), and Rein et al. (2015). 
Table 3. Eigenvalues, percentage variance by axis, and cumulative percentage of variance for the principal component analysis of entire sample.

\begin{tabular}{|c|c|c|c|}
\hline Axis & Eigenvalues & $\%$ Variance & Cumulative \% \\
\hline 1 & 0.00039695 & 44.496 & 44.496 \\
\hline 2 & 0.00016072 & 18.016 & 62.512 \\
\hline 3 & 0.00007785 & 8.726 & 71.238 \\
\hline 4 & 0.0000367 & 4.113 & 75.351 \\
\hline 5 & 0.00002666 & 2.988 & 78.340 \\
\hline 6 & 0.0000202 & 2.264 & 80.604 \\
\hline 7 & 0.00001921 & 2.154 & 82.758 \\
\hline 8 & 0.00001759 & 1.972 & 84.730 \\
\hline 9 & 0.00001421 & 1.593 & 86.322 \\
\hline 10 & 0.00001123 & 1.259 & 87.581 \\
\hline 11 & 0.00000935 & 1.048 & 88.629 \\
\hline 12 & 0.00000856 & 0.959 & 89.588 \\
\hline 13 & 0.00000706 & 0.792 & 90.380 \\
\hline 14 & 0.00000676 & 0.757 & 91.137 \\
\hline 15 & 0.00000635 & 0.712 & 91.849 \\
\hline 16 & 0.00000604 & 0.677 & 92.526 \\
\hline 17 & 0.00000555 & 0.622 & 93.148 \\
\hline 18 & 0.00000501 & 0.561 & 93.709 \\
\hline 19 & 0.00000496 & 0.556 & 94.265 \\
\hline 20 & 0.0000045 & 0.505 & 94.769 \\
\hline
\end{tabular}




\begin{tabular}{|c|c|c|c|}
\hline 21 & 0.0000041 & 0.459 & 95.229 \\
\hline 22 & 0.00000384 & 0.430 & 95.659 \\
\hline 23 & 0.00000347 & 0.389 & 96.048 \\
\hline 24 & 0.00000325 & 0.364 & 96.411 \\
\hline 25 & 0.00000309 & 0.347 & 96.758 \\
\hline 26 & 0.00000284 & 0.318 & 97.076 \\
\hline 27 & 0.00000249 & 0.279 & 97.356 \\
\hline 28 & 0.00000232 & 0.260 & 97.616 \\
\hline 29 & 0.00000212 & 0.238 & 97.854 \\
\hline 30 & 0.00000191 & 0.215 & 98.068 \\
\hline 31 & 0.00000187 & 0.209 & 98.278 \\
\hline 32 & 0.00000183 & 0.205 & 98.483 \\
\hline 33 & 0.00000169 & 0.190 & 98.673 \\
\hline 34 & 0.00000154 & 0.173 & 98.846 \\
\hline 35 & 0.00000152 & 0.170 & 99.016 \\
\hline 36 & 0.00000141 & 0.158 & 99.174 \\
\hline 37 & 0.00000134 & 0.150 & 99.324 \\
\hline 38 & 0.00000118 & 0.132 & 99.457 \\
\hline 39 & 0.00000114 & 0.127 & 99.584 \\
\hline 40 & 0.000001 & 0.112 & 99.696 \\
\hline 41 & 0.00000078 & 0.088 & 99.784 \\
\hline 42 & 0.00000073 & 0.082 & 99.866 \\
\hline 43 & 0.00000067 & 0.075 & 99.940 \\
\hline
\end{tabular}


Table 4. Eigenvalues, percentage variance by axis, and cumulative percentage of variance for the principal component analysis of species mean configurations.

\begin{tabular}{|c|c|c|c|}
\hline Axis & Eigenvalues & $\%$ Variance & Cumulative \% \\
\hline 1 & 0.00035069 & 53.840 & 53.840 \\
\hline 2 & 0.00013535 & 20.780 & 74.620 \\
\hline 3 & 0.00006617 & 10.158 & 84.779 \\
\hline 4 & 0.00003104 & 4.765 & 89.544 \\
\hline 5 & 0.00001425 & 2.187 & 91.731 \\
\hline 6 & 0.00001329 & 2.040 & 93.771 \\
\hline 7 & 0.00000928 & 1.425 & 95.196 \\
\hline 8 & 0.00000769 & 1.180 & 96.376 \\
\hline 9 & 0.00000638 & 0.979 & 97.356 \\
\hline 10 & 0.00000458 & 0.703 & 98.059 \\
\hline 11 & 0.0000028 & 0.430 & 98.490 \\
\hline 12 & 0.0000022 & 0.338 & 98.828 \\
\hline 13 & 0.00000199 & 0.306 & 99.134 \\
\hline 14 & 0.00000161 & 0.247 & 99.381 \\
\hline 15 & 0.00000123 & 0.189 & 99.569 \\
\hline 16 & 0.00000106 & 0.162 & 99.732 \\
\hline 17 & 0.0000006 & 0.091 & 99.823 \\
\hline 18 & 0.00000048 & 0.074 & 99.897 \\
\hline 19 & 0.00000037 & 0.057 & 99.954 \\
\hline 20 & 0.0000003 & 0.046 & 100.000 \\
\hline
\end{tabular}


Table 5. Regression coefficients for the pGLS model based on Pagel's lambda ${ }^{a}$

\begin{tabular}{|c|c|c|c|c|c|}
\hline & Independent & & & & \\
\hline PC score & Variable & Coef & SE & $p$ & Pagel's $\lambda$ \\
\hline \multirow[t]{2}{*}{ One } & $\log _{10}(\mathrm{CS})$ & 0.027 & 0.009 & $<0.01$ & 0.96 \\
\hline & $\operatorname{logit}(\mathrm{FSL})$ & -0.011 & 0.002 & 0.0001 & \\
\hline \multirow[t]{2}{*}{ One } & $\ln ($ Ulna SS $)$ & 0.029 & 0.006 & $<0.001$ & 0.91 \\
\hline & $\operatorname{logit}(\mathrm{FSL})$ & -0.007 & 0.002 & $<0.001$ & \\
\hline \multirow[t]{2}{*}{ Two } & $\log _{10}(\mathrm{CS})$ & -0.015 & 0.002 & $<0.0001$ & 0.35 \\
\hline & $\operatorname{logit}(\mathrm{FSL})$ & -0.006 & 0.001 & $<0.0001$ & \\
\hline \multirow[t]{2}{*}{ Two } & $\ln ($ Ulna SS $)$ & -0.011 & 0.003 & $<0.001$ & 0.54 \\
\hline & $\operatorname{logit}(\mathrm{FSL})$ & -0.007 & 0.001 & $<0.0001$ & \\
\hline
\end{tabular}

${ }^{\mathrm{a}}$ Abbreviations: $\mathrm{pGLS}=$ phylogenetic generalized least-squares, $\mathrm{PC}=$ principal component, Coef = coefficient, $\mathrm{SE}=$ standard error, $\mathrm{CS}=$ centroid size, $\mathrm{FSL}=$ forelimb suspensory locomotion proportion, $\mathrm{SS}=$ size surrogate. Pagel's lambda based on restricted maximum likelihood estimate. 
Table 6. Regression coefficients for multiple regressions of principal component (PC) scores against linear measurement shape variables ${ }^{\mathrm{a}}$

\begin{tabular}{|c|c|c|c|c|}
\hline & Independent & & & \\
\hline PC score & Variable & Coef & SE & $p$ \\
\hline \multirow[t]{3}{*}{ One } & AP/PD RAD & 0.02 & 0.02 & 0.31 \\
\hline & $\ln (\mathrm{UL})$ & -0.04 & 0.01 & $<0.01$ \\
\hline & $\ln (\mathrm{Ulna} \mathrm{SS})$ & 0.08 & 0.01 & $<0.0001$ \\
\hline \multirow[t]{3}{*}{ One } & PD/ML TROCH & -0.02 & 0.02 & 0.17 \\
\hline & $\ln (\mathrm{UL})$ & -0.04 & 0.01 & $<0.01$ \\
\hline & $\ln (\mathrm{Ulna} \mathrm{SS})$ & 0.07 & 0.01 & $<0.0001$ \\
\hline \multirow[t]{3}{*}{ Two } & AP/PD RAD & 0.02 & 0.01 & 0.01 \\
\hline & $\ln (\mathrm{UL})$ & -0.05 & 0.005 & $<0.0001$ \\
\hline & $\ln (\mathrm{Ulna} \mathrm{SS})$ & 0.03 & 0.005 & $<0.0001$ \\
\hline \multirow[t]{3}{*}{ Two } & PD/ML TROCH & 0.02 & 0.01 & $<0.01$ \\
\hline & $\ln (\mathrm{UL})$ & -0.05 & 0.004 & $<0.0001$ \\
\hline & ln(Ulna SS) & 0.03 & 0.005 & $<0.0001$ \\
\hline
\end{tabular}

${ }^{\mathrm{a}}$ Abbreviations: Coef $=$ coefficient, $\mathrm{SE}=$ standard error, $\mathrm{AP} / \mathrm{PD} \mathrm{RAD}=$ ratio of anteroposterior and proximodistal radial notch lengths, $\mathrm{UL}=$ ulnar length, $\mathrm{SS}=$ size surrogate, $\mathrm{PD} / \mathrm{ML} \mathrm{TROCH}$ $=$ ratio of proximodistal and mediolateral lengths of trochlear notch. 
Table 7. Regression coefficients for multiple regressions of forelimb suspensory locomotion (FSL) against principal component (PC) scores and linear measurement variables ${ }^{\mathrm{a}}$

$$
\text { Independent }
$$

\begin{tabular}{lcccc} 
Dependent & Variable & Coef & SE & $p$ \\
\hline FSL & PC 1 & -30.0 & 5.4 & $<0.0001$ \\
$\mathrm{R}^{2}=0.92$ & & & & \\
$\mathrm{RSE}=0.4$ & PC 2 & -100.8 & 8.5 & $<0.0001$ \\
FSL & $\ln (\mathrm{UL})$ & 6.1 & 1.0 & $<0.0001$ \\
$\mathrm{R}^{2}=0.70$ & & & & \\
$\mathrm{RSE}=0.8$ & $\ln (\mathrm{Ulna} S \mathrm{SS})$ & -5.1 & 1.0 & $<0.001$ \\
\hline
\end{tabular}

${ }^{\mathrm{a}}$ Abbreviations: Coef $=$ coefficient, $\mathrm{SE}=$ standard error, $\mathrm{RSE}=$ residual standard error, $\mathrm{UL}=$ ulnar length, Ulna SS = ulnar size surrogate. 
Table 8. Procrustes distances between mean species configurations of the Hominidae ${ }^{\mathrm{a}}$

$$
\text { Gorilla Homo Pan Pongo A. afarensis A. sediba }
$$

Gorilla $\quad 0.0000$

Homo $\quad 0.0244 \quad 0.0000$

Pan $\quad 0.0316 \quad 0.0199 \quad 0.0000$

$\begin{array}{lllll}\text { Pongo } & 0.0432 & 0.0359 & 0.0269 & 0.0000\end{array}$

$\begin{array}{llllll}\text { A. afarensis } & 0.0365 & 0.0277 & 0.0230 & 0.0345 & 0.0000\end{array}$

$\begin{array}{lllllll}\text { A. sediba } & 0.0440 & 0.0355 & 0.0255 & 0.0268 & 0.0268 & 0.0000\end{array}$

${ }^{a}$ Hominidae refers to great apes and hominins 


$$
11: 11
$$


Figure 2

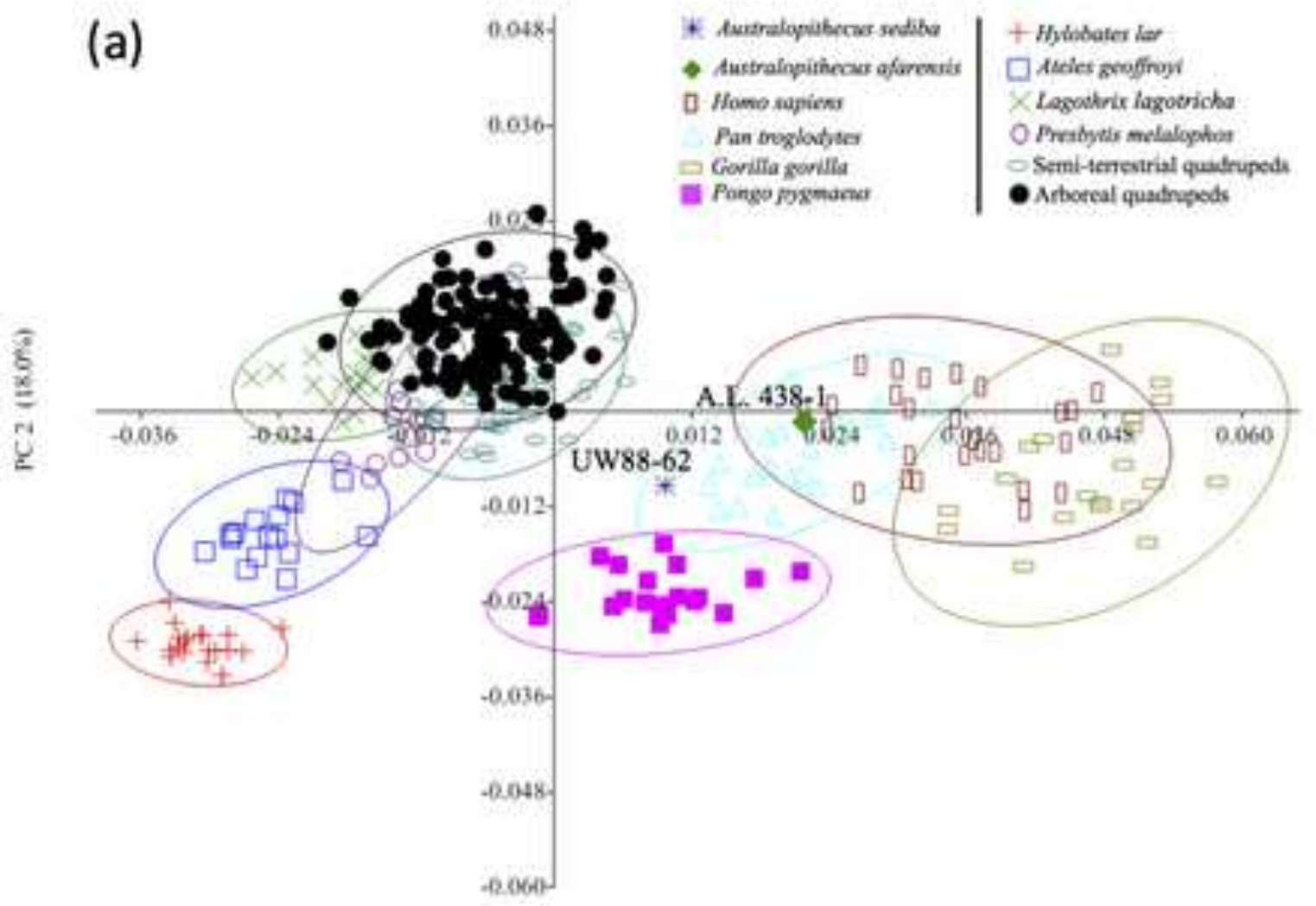

PC $1(445 \%)$

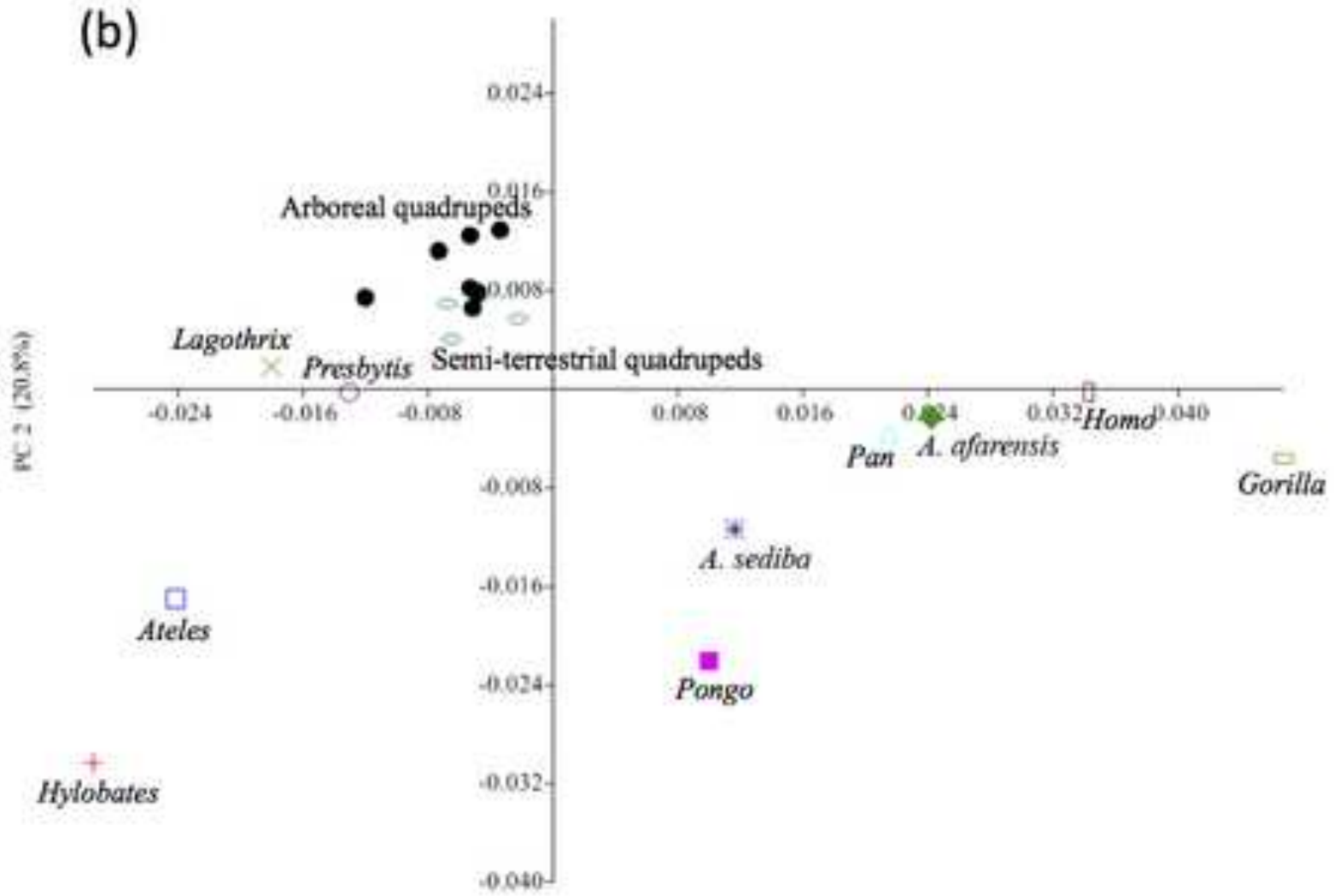

PC 1 (53.8\%) 

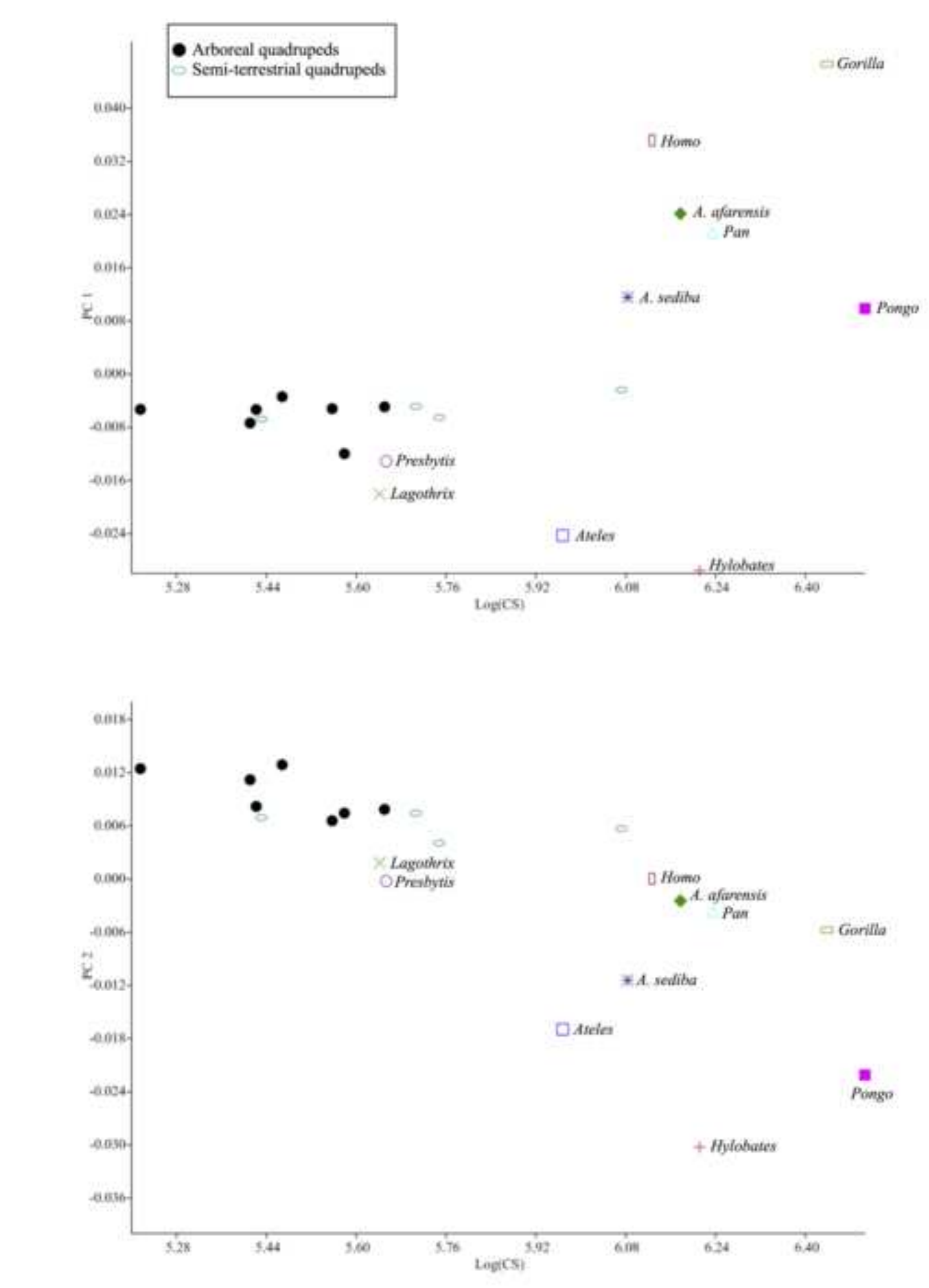

Figure 3

3

\section{Figure 3}

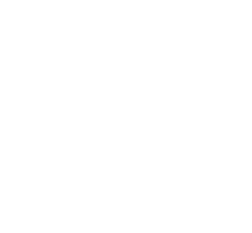

-

-

(
.

$\sqrt{2}+x^{2}$

$+2$


Figure 4

\section{$-0.04$

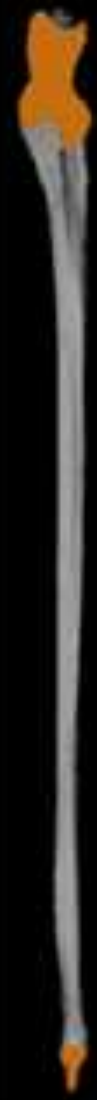

PC 10.06

$-0.04$

PC 20.04
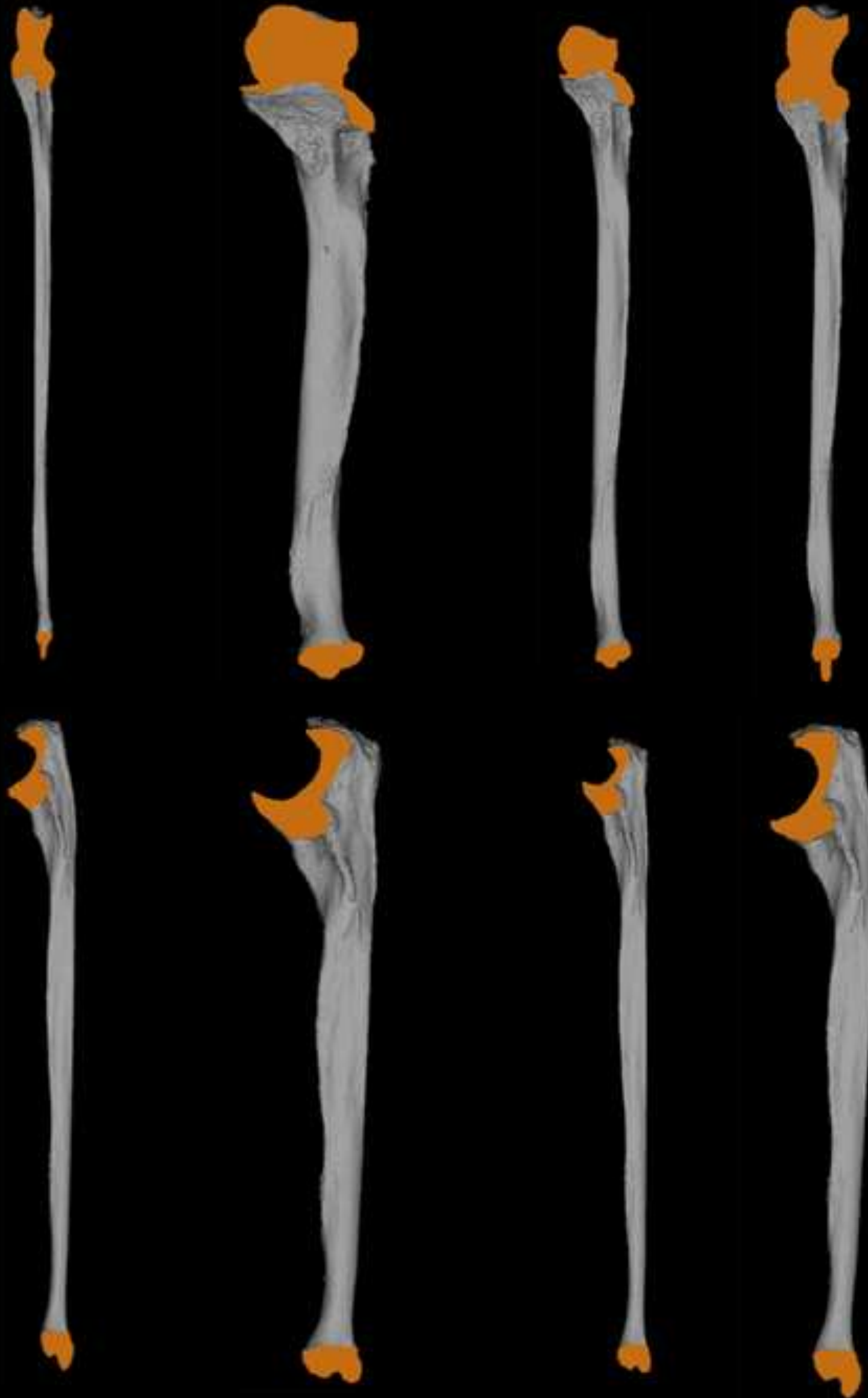


\section{Figure 5}
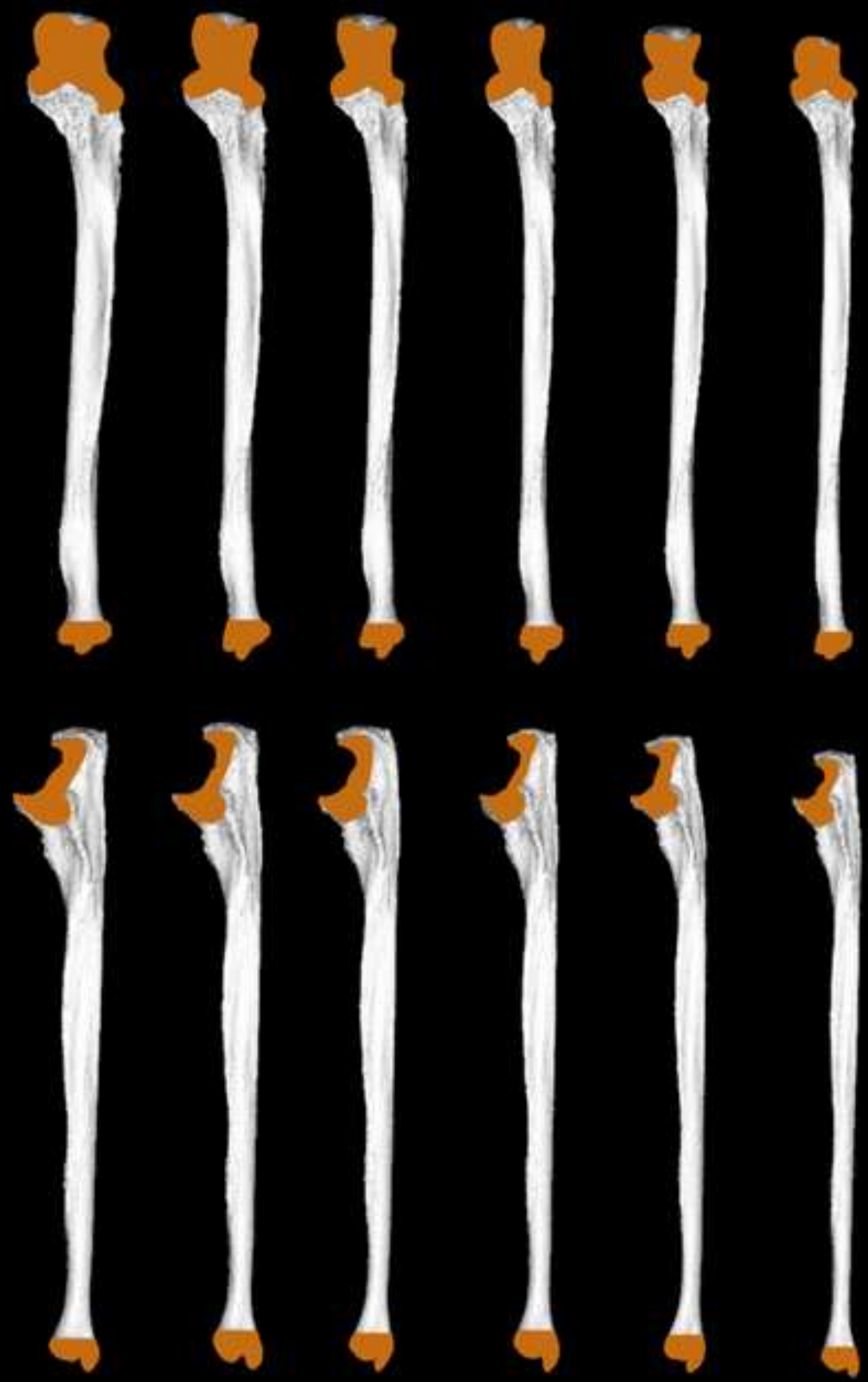

(a)

(b)

(c)

(d)

(e)

(f) 


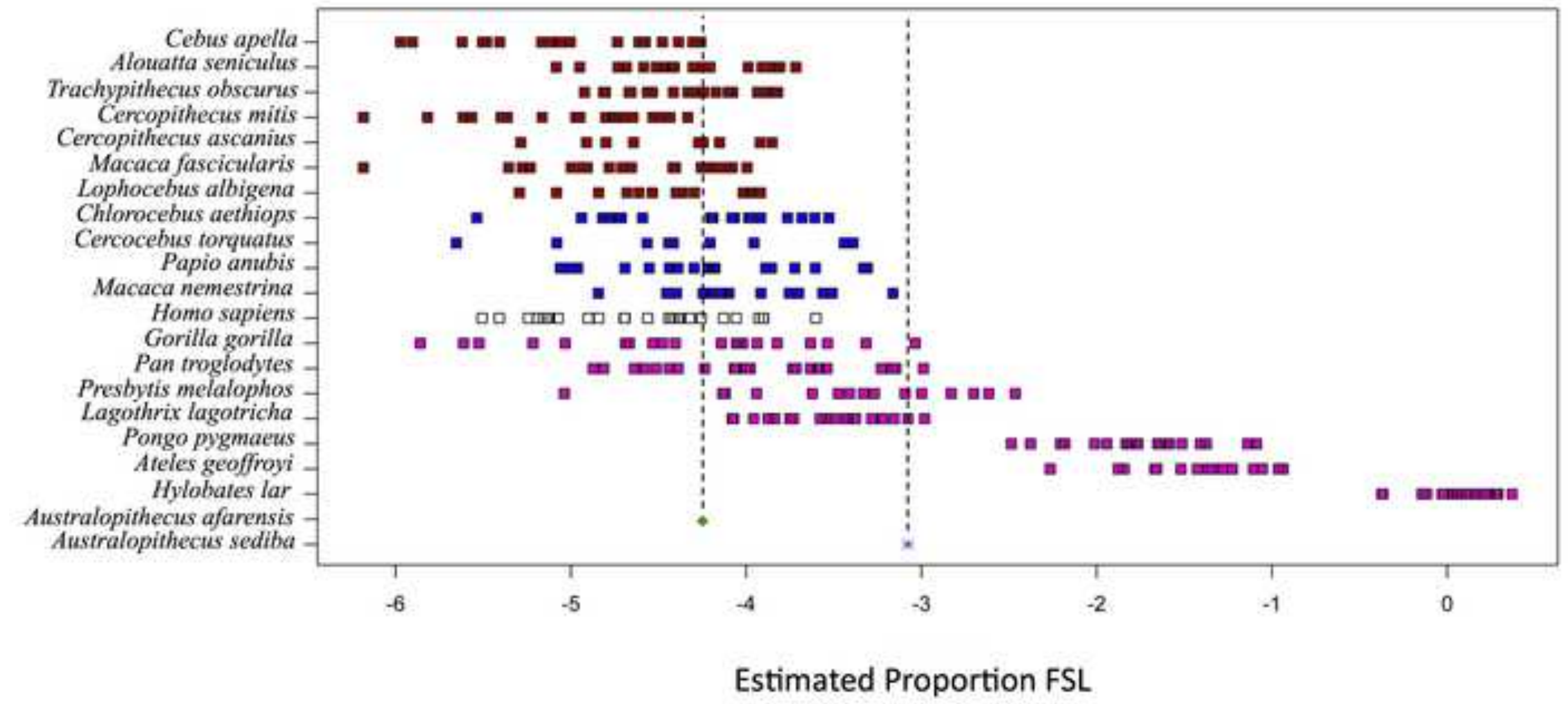

